Note

\title{
The solar Poynting-Robertson effect on particles orbiting Solar System bodies: Circular orbits
}

\author{
David Parry Rubincam* \\ Planetary Geodynamics Laboratory, Code 698, Solar System Exploration Division, NASA Goddard Space Flight Center, Building 34, Room S280, Greenbelt, MD 20771, United States
}

\section{A R T I C L E I N F O}

\section{Article history:}

Received 9 April 2013

Revised 24 July 2013

Accepted 25 July 2013

Available online 6 August 2013

\section{Keywords:}

Near-Earth objects

Interplanetary dust

Celestial mechanics

Planetary rings

Saturn, Rings

\begin{abstract}
A B S T R A C T
The Poynting-Robertson effect from sunlight impinging directly on a particle which orbits a Solar System body (planet, asteroid, comet) is considered from the Sun's rest frame. There appear to be no significant first-order terms in $V_{b} / c$ for circular orbits, where $V_{b}$ is the body's speed in its orbit about the Sun and $c$ is the speed of light, when the particle's orbital semimajor axis is much smaller than the body's orbital semimajor axis about the Sun as is mainly the case in the Solar System.
\end{abstract}

Published by Elsevier Inc.

\section{Introduction}

The impetus for the current paper is the environment around NEAs (Near-Earth Asteroids). Debris might be thrown off a solitary NEA via Yarkovsky-O'Keefe-Radzieevski-Paddack (YORP) spin-up (Paddack, 1969; Rubincam, 2000), from the fission of an NEA into a binary system (e.g., Jacobson and Scheeres, 2011), from tidal encounters with planets (e.g., Richardson et al., 1998), or from impacts. Debris orbiting around an NEA could be a factor in the safety of a manned mission to one of these tiny asteroids. Hence understanding the forces on possible debris is desirable.

Orbital debris will be acted upon by radiation forces (photon thrust) as well as by gravitation. Solar radiation pressure and the Poynting-Robertson effect are the best known of all the radiation forces. Poynting-Robertson is a $V / c$ effect (where $V$ is the speed and $c$ is the speed of light); it causes orbit decay for dust or ice particles orbiting a luminous body (e.g., Poynting, 1903; Robertson, 1937; Burns et al., 1979). Being a surface-to-mass effect, the smaller the particle, the faster the decay in the geometric optics limit. Poynting-Robertson drag will act on Earth satellites as well, though at a much slower pace than on ice or dust particles, because of the unfavorable surface-to-mass ratio.

The Poynting-Robertson effect is most commonly applied to small particles orbiting the Sun, but it also applies to particles orbiting planets (e.g., Burns et al., 1979; Goldreich and Tremaine, 1982; Mignard, 1984; Burns et al., 1984, 1999; Vokrouhlický

\footnotetext{
* Fax: +1 3016146522 .

E-mail address: David.P.Rubincam@nasa.gov
}

et al., 2007), as well as moons, comets, and asteroids, since these all reflect visible light and emit in the infrared. Complications arise in computing Poynting-Robertson drag for orbits around these objects, because the body being orbited is not the only source of photons; the Sun shines on the particles as well. The sunshine ceases when a particle enters the body's shadow, and both the sunshine and its cessation must be dealt with. What happens when the particle orbit intersects the body's shadow is less clear.

Vokrouhlický et al. (2007, on-line Appendix B) investigated the solar Poynting-Robertson effect in Saturn's icy rings and concluded that $V_{b} / c$ enters into the calculation at a significant level, where $V_{b}$ is the speed of the body in its orbit about the Sun. They found that the Poynting-Robertson force on a particle from the solar photons does not average out when integrating around its orbit when the orbit intersects Saturn's shadow. Usually only the order-of-magnitude of the particle's orbital decay rate is desired, so that for particles orbiting about Saturn or Jupiter with speed $v, V_{b} \sim v$ (Table 1 ), and whether $V_{b}$ enters the calculation or not would not make a big difference in the orbital decay rate and is therefore not particularly important.

However, the situation changes when considering a particle orbiting an NEA. In this case the NEA's mass is small, so that $v$ might be on the order of $\sim 1 \mathrm{~m} \mathrm{~s}^{-1}$, while $V_{b}=\sim 3 \times 10^{4} \mathrm{~m} \mathrm{~s}^{-1}$ if the asteroid is in an Earth-like orbit (Table 1). Hence $V_{b} \gg v$ and shadow passage could be highly important for the Poynting-Robertson effect when considering particles orbiting NEAs if $V_{b}$ indeed enters into the Poynting-Robertson drag at a significant level.

The present paper works the problem of what happens to the Poynting-Robertson drag when a particle in a circular orbit about 
Table 1

Parameters for six planets and two asteroids.

\begin{tabular}{lllllll}
\hline Body & $\begin{array}{l}V_{b}{ }^{\mathrm{a}} \\
\left(10^{3} \mathrm{~m} \mathrm{~s}^{-1}\right)\end{array}$ & $\begin{array}{l}V_{b} / c^{\mathrm{b}} \\
\left(10^{-5}\right)\end{array}$ & $\begin{array}{l}R^{\mathrm{c}} / d^{\mathrm{d}} \\
\left(10^{-5}\right)\end{array}$ & $\begin{array}{l}v^{\mathrm{e}} \\
\left(10^{3} \mathrm{~m} \mathrm{~s}^{-1}\right)\end{array}$ & $\begin{array}{l}v / c \\
\left(10^{-5}\right)\end{array}$ & $V_{b} / v$ \\
\hline Mercury & 47.62 & 15.87 & 4.17 & 3.00 & 1.00 & 15.87 \\
Venus & 35.05 & 11.68 & 5.60 & 7.33 & 2.44 & 4.78 \\
Earth & 29.74 & 9.91 & 4.26 & 7.90 & 2.63 & 3.76 \\
Mars & 24.12 & 8.04 & 1.49 & 3.55 & 1.18 & 6.79 \\
Jupiter & 13.04 & 4.35 & 9.17 & 42.09 & 14.03 & 0.31 \\
Saturn & 9.61 & 3.20 & 4.20 & 25.08 & 8.36 & 0.38 \\
NEA $^{\mathrm{f}}$ & 29.74 & 9.91 & 0.0007 & 0.0007 & 0.0003 & $3 \times 10^{4}$ \\
Ida & 17.59 & 5.86 & 0.004 & 0.013 & 0.004 & $1.4 \times 10^{4}$ \\
\hline
\end{tabular}

a Speed of the body about the Sun.

b Speed of light

c Radius of the body.

d Distance of the particle from the Sun.

e Speed around the body for $r=R$.

f Hypothetical asteroid with radius $R=10^{3} \mathrm{~m}$, density $=2 \times 10^{3} \mathrm{~kg} \mathrm{~m}^{-3}$, and in Earth's orbit.

a spherical Solar System body intersects the body's shadow and the Sun's light is cut off. The resultant equations indicate that $V_{b}$ is not nearly as important as found by Vokrouhlický et al. (2007). The reason is that, even though the solar photons emanate from the solar direction, the shadow does not point directly away from the Sun when viewed from the Sun's rest frame. A body in a circular orbit about the Sun travels at a speed $V_{b}$ at right angles to a field of photons traveling at a speed $c$, so that there is the aberration of starlight, with the star being the Sun. The body's shadow is thus rotated slightly. This slight rotation causes the direct solar radiation pressure on the particle to act in such a way as to cancel to order $V_{b} / c$ the solar Poynting-Robertson effect from the sunlit portion of the orbit.

\section{Circular coplanar orbits}

Consider a spherical particle of mass $M_{p}$ and radius $R_{p}$ at a distance $d$ from the Sun. Burns et al. (1979) in their Eq. (2) show that the acceleration in the Sun's inertial rest frame due to solar radiation pressure from the Sun is given by

$\mathbf{f}_{\text {press }}=\frac{B}{d^{2}} \hat{\mathbf{d}}$

and the Poynting-Robertson acceleration $\mathbf{f}_{\mathbf{P R}}$ from the Sun is

$\mathbf{f}_{\mathbf{P R}}=\frac{B}{d^{2}}\left[-\frac{\mathbf{V}}{c}-\left(\frac{\mathbf{V} \cdot \hat{\mathbf{d}}}{c}\right) \hat{\mathbf{d}}\right]=\frac{B}{d^{2}}\left[-\frac{\mathbf{V}}{c}-\left(\frac{\mathbf{V} \cdot \mathbf{d}}{d^{2} c}\right) \mathbf{d}\right]$

where $\mathbf{V}$ is the particle's speed in the Sun's rest frame, $\hat{\mathbf{d}}$ is the unit vector from the Sun to the particle (karats (hats) over quantities signify unit vectors), and $\mathbf{d}=d \hat{\mathbf{d}}$ is the particle's position in the Sun's frame. Also,

$B=\frac{\pi Q R_{p}^{2} F_{E} a_{E}^{2}}{M_{p} c}$

where $c$ is the speed of light, $Q$ is a factor which depends on the way the particle absorbs and emits light, and $F_{E}=1361 \mathrm{~J} \mathrm{~m}^{-2} \mathrm{~s}^{-1}$ is the insolation at the Earth's distance $a_{E}=1 \mathrm{AU}$ from the Sun (Kopp and Lean, 2011).

Eqs. (1)-(3) are general. The simplest qualitative explanation for the null result relies on considering the particular case of coplanar orbits (Fig. 1). The body is assumed to be spherical and at the center of the $(x, y, z)$ coordinate system; the $x$-axis points down and the $y$-axis points to the right. The Sun is assumed to be at rest in an inertial coordinate system and is a point-source of light on the $-y$ axis far enough away from the body so that the Sun's rays

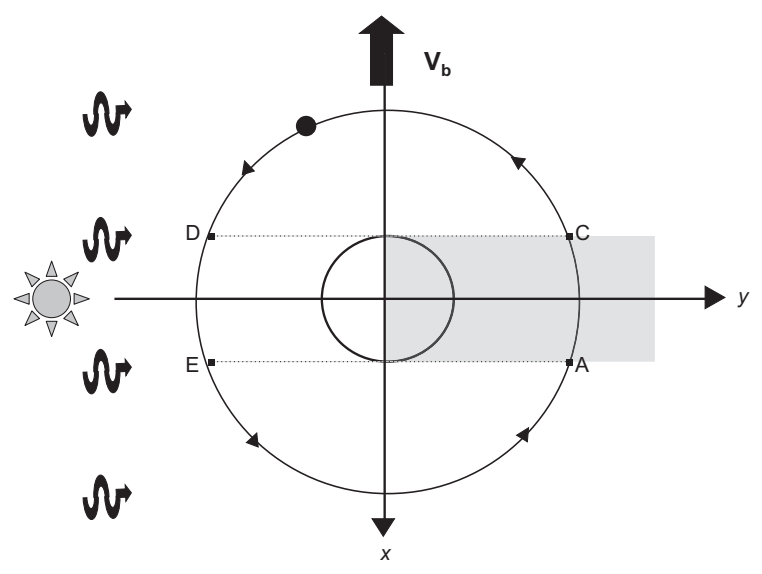

Fig. 1. Schematic geometry of coplanar orbits as seen from the Sun's rest frame, assuming no rotation of shadow. A particle (black dot) moves around a spherical body in a circular orbit. The body orbits the Sun in a circular orbit with orbital velocity $\mathbf{V}_{\mathbf{b}}$ (large black arrow) in the $-x$ direction. The Sun is on the $-y$ axis, so that its rays come in from the left (wavy arrows). The part of the Poynting-Robertson force which is proportional to $-\mathbf{V}_{\mathbf{b}} / \mathbf{c}$ on arc $\mathrm{DE}$ is not canceled out at arc AC because of the shadow.

travel on parallel trajectories (wavy arrows). The system travels with a speed $V_{b}$ in the $-x$ direction. In the particular case considered in Fig. 1, a spherical particle (black dot) happens to move in its circular orbit in the positive sense; moving in the opposite sense is discussed later.

The position in the Sun's frame of a particle orbiting a Solar System body can be written

$\mathbf{d}=d \hat{\mathbf{d}}=\mathbf{r}_{\mathbf{b}}+\mathbf{r}$

where $\mathbf{r}_{\mathbf{b}}$ is the vector from the Sun's center to the body's center, and $\mathbf{r}$ is the vector from the body's center to the particle. The particle's velocity in the Sun's rest frame is then

$\dot{\mathbf{d}}=\dot{\mathbf{r}}_{\mathbf{b}}+\dot{\mathbf{r}}=\mathbf{V}_{\mathbf{b}}+\mathbf{v}$

where $\mathbf{V}_{\mathbf{b}}$ is the body's velocity in the Sun's frame, with $\left|\mathbf{V}_{\mathbf{b}}\right|=V_{b}$, and $\mathbf{v}$ is the velocity of the particle in the $(x, y, z)$ frame of the body, with $|\mathbf{v}|=v$. The distance $d$ from the Sun to the particle can be written

$d=r_{b}\left(1+\frac{2 \mathbf{r}_{\mathbf{b}} \cdot \mathbf{r}}{r_{b}^{2}}+\frac{r^{2}}{r_{b}^{2}}\right)^{1 / 2}$

where $\left|\mathbf{r}_{\mathbf{b}}\right|=r_{b}$ and $|\mathbf{r}|=r$. Using (4) and (5) in (2) allows the Poynting-Robertson acceleration to be written

$$
\begin{aligned}
\mathbf{f}_{\mathrm{PR}} & =\left(\frac{B}{d^{2}}\right)\left[-\frac{\mathbf{V}_{\mathbf{b}}}{c}-\left(\frac{\mathbf{V}_{\mathbf{b}} \cdot \mathbf{r}_{\mathbf{b}}}{d^{2} c}\right) \mathbf{d}-\left(\frac{\mathbf{V}_{\mathbf{b}} \cdot \mathbf{r}}{d^{2} c}\right) \mathbf{d}-\frac{\mathbf{v}}{c}-\left(\frac{\mathbf{v} \cdot \mathbf{r}_{\mathbf{b}}}{d^{2} c}\right) \mathbf{d}-\left(\frac{\mathbf{v} \cdot \mathbf{r}}{d^{2} c}\right) \mathbf{d}\right] \\
& =\mathbf{f}_{\mathrm{PR} 1}+\mathbf{f}_{\mathbf{P R} 2}+\mathbf{f}_{\mathrm{PR} 3}+\mathbf{f}_{\mathrm{PR} 4}+\mathbf{f}_{\mathrm{PR} 5}+\mathbf{f}_{\mathrm{PR} 6}
\end{aligned}
$$

where

$\mathbf{f}_{\mathbf{P R} 1}=-\left(\frac{B}{d^{2}}\right)\left(\frac{\mathbf{V}_{\mathbf{b}}}{c}\right)$

etc., correspond to the various terms in (7).

Consider qualitatively the role of (8) in the particle's motion. Viewed from the Sun's inertial frame, it would appear from Fig. 1 that $-\mathbf{V}_{\mathbf{b}} / c$ should contribute to the Poynting-Robertson drag when averaging the force around the orbit. If the shadow (gray region) points directly away from the Sun, then the body's umbra is a cylinder whose axis stretches along the positive $y$-axis as shown in the figure. The shadowed portion of the orbit is confined between 
the horizontal dotted lines. The particle enters the shadow at A and exits at $C$. The force on the portion of the orbit between points $D$ and $\mathrm{E}$ is not canceled out by the shadowed portion between $\mathrm{A}$ and $\mathrm{C}$, and so a net contribution to the force should result from (8). This force would actually be a boost instead of a drag because the Poynting-Robertson force acts in the direction opposite to $\mathbf{V}_{\mathbf{b}}$; between $\mathrm{D}$ and $\mathrm{E}$ the particle is pushed along in its orbit, according to (8). (See also Vokrouhlický et al., 2007, second term in B.11.)

However, viewed from the $(x, y, z)$ frame of the body, $\mathbf{V}_{\mathbf{b}}$ vanishes and its only effect is the Sun apparently shifting its position due to the aberration of starlight (Fig. 2), so that there is no $\mathbf{V}_{\mathbf{b}} / \mathbf{c}$ contribution to Poynting-Robertson. Thus there seems to be a contradiction: the results differ, with $\mathbf{V}_{\mathbf{b}} / c$ appearing or not, depending upon which frame is chosen.

The resolution of the contradiction lies in examining what happens in the Sun's rest frame more closely (Fig. 3). Because the system is traveling at speed $V_{b}$, the shadow (gray region) is rotated by an angle $\alpha$, where

$\tan \alpha=V_{b} / c \approx \alpha$

with respect to the $y$-axis due to the aberration of starlight (e.g., Sommerfeld, 1964, pp. 63-66). A fuller treatment of the shadow rotation is given in the Appendix. The particle gets a Poynting-Robertson boost between $\mathrm{G}$ and $\mathrm{H}$ from (8). It would also have gotten a boost between $\mathrm{F}$ and $\mathrm{A}$ from solar radiation pressure (wavy arrows), except now the shadow is there and the radiation pressure vanishes between $\mathrm{F}$ and $\mathrm{A}$. Between $\mathrm{B}$ and $\mathrm{C}$ direct solar radiation pressure (given by (1)) acts against the motion. It will be shown quantitatively below that these combine to cancel the Poynting-Robertson boost at $\mathrm{GH}$, leaving a null result to order $V_{b} / c$ for circular coplanar orbits.

There is also a null result if the particle orbits in the sense opposite to that shown in Fig. 3. In that case there would now be Poynting-Robertson drag on the arc HG, but there is a solar radiation pressure boost between $\mathrm{CB}$, and again nothing at $\mathrm{AF}$, once more giving a null result. Hence there is no net effect regardless of the sense of motion around the body. (While eccentric orbits are not treated here, the semimajor axis of eccentric orbits will change as particles move at an angle through the shadow. However, the effect is periodic due to the precession of pericenter and so are unimportant in the long run. See Mignard (1984) and Horanyi and Burns (1991).)

What follows next is a quantitative proof of a null result to order $V_{b} / c$ for an inclined circular particle orbit when (8) combines with (1). The coplanar orbits discussed above are simply a special

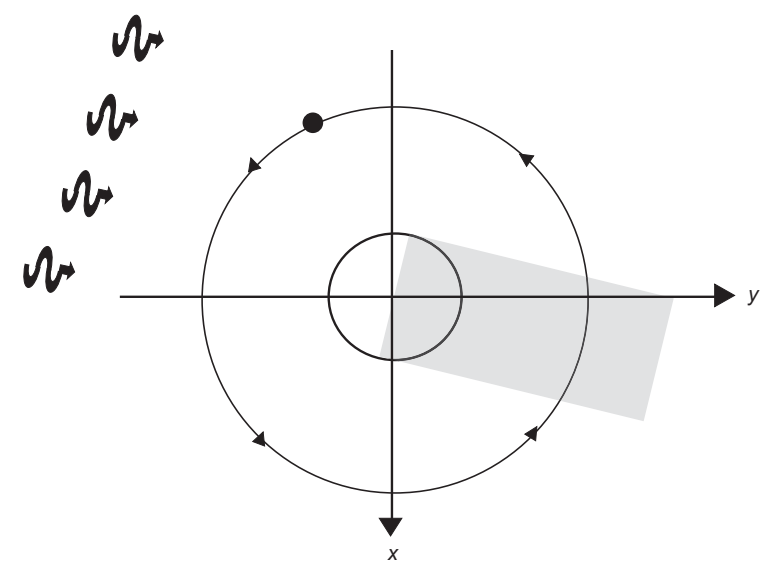

Fig. 2. The orbits as seen in the body's frame. The Sun's position appears shifted by an angle $\sim V_{b} / c$ because of the aberration of starlight, and there is no net PoyntingRobertson effect which is proportional to $-\mathbf{V}_{\mathbf{b}} / c$.

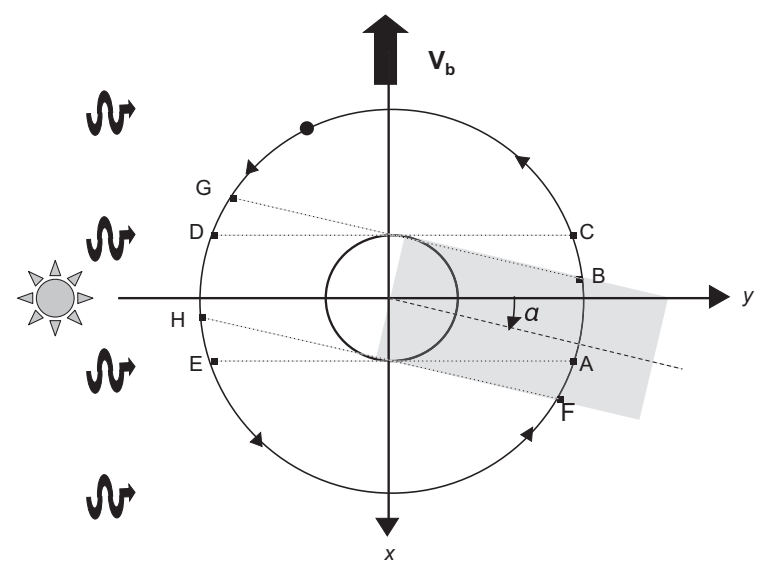

Fig. 3. Coplanar orbits seen from the Sun's rest frame as in Fig. 1, this time with the shadow rotated due to the aberration of starlight. The radiation pressure on $\mathrm{BC}$ and its lack on FA cancels out the $-\mathbf{V}_{\mathbf{b}} / c$ Poynting-Robertson effect on $\mathrm{GH}$.

case where the inclination is zero. The other terms in (7) are discussed later.

\section{Inclined circular orbit}

The geometry of the particle's inclined orbit is shown in Fig. 4. Once again the Sun is on the $-y$ axis and the coordinate system travels in the $-x$ direction. The orbit is now inclined by the angle $I$ to the $z$-axis and the unit vector

$\hat{\mathbf{n}}=(\sin I \sin \Omega) \hat{\mathbf{x}}-(\sin I \cos \Omega) \hat{\mathbf{y}}+(\cos I) \hat{\mathbf{z}}$

is normal to the orbital plane, where $\hat{\mathbf{x}}, \hat{\mathbf{y}}$, and $\hat{\mathbf{z}}$ are unit vectors along the respective axes. The angle in the $x-y$ plane of the ascending node is given by $\Omega$, while the argument of pericenter is angle $\omega$ and the true anomaly is angle $f$. Because the orbit is assumed here to be circular, only the angle $\omega+f$ is meaningful. The axis of the cylindrical umbra lies in the $x-y$ plane and is rotated by an angle $\alpha$ from the $y$-axis because of the aberration of starlight; $\alpha$ is positive for the sense shown. Note that this is opposite to the usual convention. The unit vector along the axis of the cylinder is thus

$\hat{\mathbf{u}}=\sin \alpha \hat{\mathbf{x}}+\cos \alpha \hat{\mathbf{y}}$

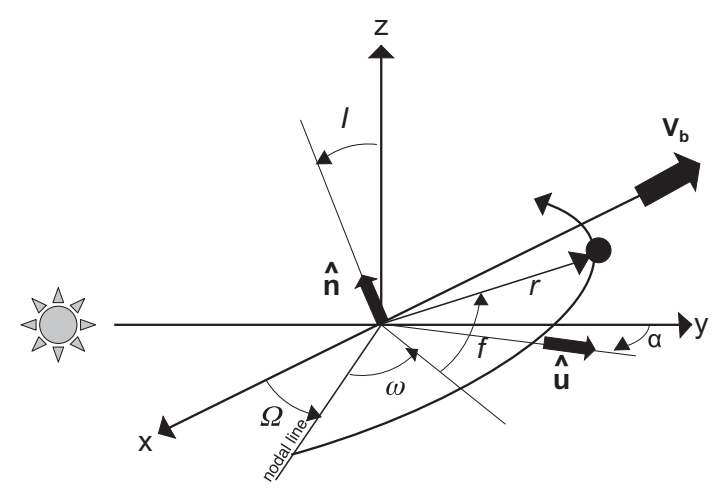

Fig. 4. The inclined particle orbit as seen from the Sun's rest frame. The particle's circumplanetary orbit is assumed to be circular. Once again the Sun is on the $-y$ axis and the $(x, y, z)$ frame travels with velocity $\mathbf{V}_{\mathbf{b}}$ in the $-x$ direction. $I$ is the inclination to the plane to the solar orbit; $\hat{\mathbf{n}}$ is the unit vector normal to the particle's orbital plane. Also, $\Omega$ is the ascending nodal angle in the $x-y$ plane, $\omega$ is the argument of pericenter, and $f$ is the true anomaly. Only the sum $\omega+f$ is meaningful for the circular orbit considered here. The axis of the umbra is rotated from the $y$-axis by an angle $\alpha$ in the $x-y$ plane. $\hat{\mathbf{u}}$ is the unit vector along the umbra's axis. 
The intersection of the particle's orbit plane with the cylinder of the umbra is half an ellipse, with the unit vector in the direction of the long axis of the ellipse being given by

$\hat{\mathbf{w}}=\hat{\mathbf{n}} \times(\hat{\mathbf{u}} \times \hat{\mathbf{n}}) /|\hat{\mathbf{n}} \times(\hat{\mathbf{u}} \times \hat{\mathbf{n}})|$

where

$|\hat{\mathbf{n}} \times(\hat{\mathbf{u}} \times \hat{\mathbf{n}})|=\left[\cos ^{2} I+\sin ^{2} I \sin ^{2}(\Omega+\alpha)\right]^{1 / 2}$

Let $\psi$ be the angle between $\hat{\mathbf{w}}$ and the nodal line. Then

$\cos \psi=\hat{\mathbf{w}} \cdot(\cos \Omega \hat{\mathbf{x}}+\sin \Omega \hat{\mathbf{y}})=\frac{\sin (\Omega+\alpha)}{\left[\cos ^{2} I+\sin ^{2} I \sin ^{2}(\Omega+\alpha)\right]^{1 / 2}}$

and

$\sin \psi=\left(1-\cos ^{2} \psi\right)^{1 / 2}=\frac{\cos I \cos (\Omega+\alpha)}{\left[\cos ^{2} I+\sin ^{2} I \sin ^{2}(\Omega+\alpha)\right]^{1 / 2}}$

The shadowed portion of the orbit can be found with the aid of Fig. 5. The $x^{\prime}-y^{\prime}$ coordinate system lies in the particle's orbital plane, with the long axis of the half-ellipse (and $\hat{\mathbf{w}}$ ) lying along the $x^{\prime}$ axis. The orbit is the equation for the circle

$\left(x^{\prime}\right)^{2}+\left(y^{\prime}\right)^{2}=r^{2}$

while that of the ellipse is

$\frac{\left(x^{\prime}\right)^{2}}{h^{2}}+\frac{\left(y^{\prime}\right)^{2}}{R^{2}}=1$

where $R$ is the radius of the body and $h$ is given by

$h=\frac{R}{|\sin I \cos (\Omega+\alpha)|}$

The orbit is in shadow when $r<h$. The shadowed portion lies between $\pm \phi$ as shown in Fig. 5. Solving (11) and (12) for $\sin \phi$ $=y^{\prime} / R$ and using (13) gives

$\sin \phi=\frac{R}{r} \frac{\left[1-\left(\frac{r}{R}\right)^{2} \sin ^{2} I \cos ^{2}(\Omega+\alpha)\right]^{1 / 2}}{\left[\cos ^{2} I+\sin ^{2} I \sin ^{2}(\Omega+\alpha)\right]^{1 / 2}}$

The Poynting-Robertson acceleration $\mathbf{f}_{\mathbf{P R} 1}$ given by (8) can be written

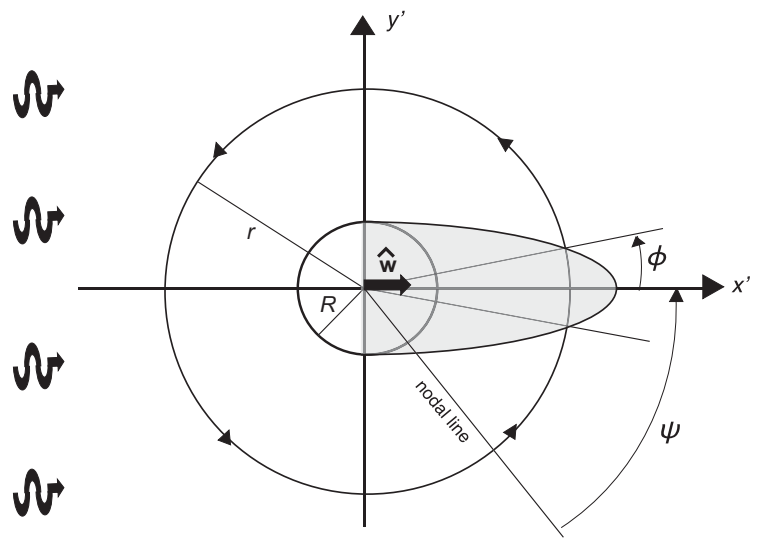

Fig. 5. The intersection of the particle's orbital $\left(x^{\prime}, y^{\prime}\right)$ plane with the umbra is half an ellipse. The ellipse's semiminor axis $R$ is the radius of the body, and the long axis lies along the $x^{\prime}$-axis. $\hat{\mathbf{w}}$ is the unit vector along the $x^{\prime}$ axis. Sunlight streams in from the left. $\psi$ Is the angle between the $x^{\prime}$ axis and the nodal line shown in Fig. 4. The circular orbit has a radius $r$ and is in shadow between $\pm \phi$ only when $r<h$.
$\mathbf{f}_{\mathrm{PR} 1}=-\frac{B}{d^{2}}\left(\frac{\mathbf{V}_{\mathbf{b}}}{c}\right) \approx+\frac{B}{r_{b}^{2}}\left(\frac{V_{b}}{c}\right) \hat{\mathbf{x}}=+\frac{B}{r_{b}^{2}} \alpha \hat{\mathbf{x}}$

where $V_{b} / c \approx \alpha$ by (9) and $d \approx r_{b}$, since in all cases of interest $V_{b} \ll c$ and $r \ll r_{b}$. The sign on the right-hand side of (15) is plus because $\mathbf{V}_{\mathbf{b}}$ is in the $-x$ direction and the force is opposite to $\mathbf{V}_{\mathbf{b}}$.

The rate of change in the semimajor axis $r$ of the circular particle orbit is given by

$\frac{d r}{d t}=\frac{2 S_{P R 1}}{n}$

(e.g., Blanco and McCuskey, 1961, p. 178), where $n$ the mean motion and $S_{P R 1}$ is the along-track acceleration. $S_{P R 1}$ is the dot-product of (15) with the along-track unit vector

$$
\begin{aligned}
\hat{\mathbf{t}}= & {[-\cos \Omega \sin (\omega+f)-\cos I \sin \Omega \cos (\omega+f)] \hat{\mathbf{x}} } \\
& +[-\sin \Omega \sin (\omega+f)+\cos I \cos \Omega \cos (\omega+f)] \hat{\mathbf{y}}+[\sin I \\
& \times \cos (\omega+f)] \hat{\mathbf{z}}
\end{aligned}
$$

(e.g., Rubincam, 1987, p. 1291). The integral of $S_{P R 1}$ averaged over one revolution of the particle orbit will be

$$
\begin{aligned}
\left\langle S_{P R 1}\right\rangle= & \alpha \frac{B}{2 \pi r_{b}^{2}} \int_{0}^{2 \pi}(\hat{\mathbf{x}} \cdot \hat{\mathbf{t}}) d(\omega+f)-\alpha \frac{B}{2 \pi r_{b}^{2}} \int_{\psi-\phi}^{\psi+\phi}(\hat{\mathbf{x}} \cdot \hat{\mathbf{t}}) \\
& d(\omega+f)
\end{aligned}
$$

assuming $\Omega$ and $I$ do not change significantly over one revolution of the particle about the body. The first integral is the acceleration assuming the whole orbit is sunlight; the second integral corrects for this by subtracting out the shadowed portion.

The first integral in (18) is 0 because integrating $\sin (\omega+f)$ and $\cos (\omega+f)$ from 0 to $2 \pi$ is zero. This leaves only the second integral. The following expressions are used in evaluating the second integral:

$\int_{\psi-\phi}^{\psi+\phi} \sin (\omega+f) d(\omega+f)=2 \sin \psi \sin \phi$

$\int_{\psi-\phi}^{\psi+\phi} \cos (\omega+f) d(\omega+f)=2 \cos \psi \sin \phi$ yields

Using these two expressions in (18) along with (15) and (17)

$$
\begin{aligned}
\left\langle S_{P R 1}\right\rangle & \approx-\alpha \frac{B}{\pi r_{b}^{2}} \int_{\psi-\phi}^{\psi+\phi}(\hat{\mathbf{x}} \cdot \hat{\mathbf{t}}) d(\omega+f) \\
& \approx+\alpha \frac{B}{\pi r_{b}^{2}} \cos I\left(\frac{R}{r}\right) \frac{\left[1-\left(\frac{r}{R}\right)^{2} \sin ^{2} I \cos ^{2} \Omega\right]^{1 / 2}}{\left[\cos ^{2} I+\sin ^{2} I \sin ^{2} \Omega\right]}
\end{aligned}
$$

to first order in $\alpha$. (See also the treatment of the shadow by Vokrouhlický et al. (2007).)

The solar radiation pressure contribution to the along-track acceleration needs to be found next. By (1) and (17) this is

$$
\begin{aligned}
& \left\langle S_{\text {press }}\right\rangle=\frac{B}{2 \pi r_{b}^{2}} \int_{0}^{2 \pi}(\hat{\mathbf{y}} \cdot \hat{\mathbf{t}}) d(\omega+f)-\frac{B}{2 \pi r_{b}^{2}} \int_{\psi-\phi}^{\psi+\phi}(\hat{\mathbf{y}} \cdot \hat{\mathbf{t}}) \\
& d(\omega+f)
\end{aligned}
$$

in (20) shows that the average along-track acceleration from solar radiation pressure turns out to be 


$$
\begin{aligned}
\left\langle S_{\text {press }}\right\rangle & \approx-\alpha \frac{B}{\pi r_{b}^{2}} \int_{\psi-\phi}^{\psi+\phi}(\hat{\mathbf{y}} \cdot \hat{\mathbf{t}}) d(\omega+f) \\
& \approx-\alpha \frac{B}{\pi r_{b}^{2}} \cos I\left(\frac{R}{r}\right) \frac{\left[1-\left(\frac{r}{R}\right)^{2} \sin ^{2} I \cos ^{2} \Omega\right]^{1 / 2}}{\left[\cos ^{2} I+\sin ^{2} I \sin ^{2} \Omega\right]}
\end{aligned}
$$

to first order in $\alpha$. Eq. (21) is the negative of (19), so that $\left\langle S_{P R 1}\right\rangle+\left\langle S_{\text {press }}\right\rangle=0$ and hence by (16) there is no net orbital evolution to first order in $V_{b} / c$ when the solar radiation pressure

(1) is combined with the Poynting-Robertson drag (8).

What about the other two terms in (7) in which $\mathbf{V}_{\mathbf{b}}$ appears? One is

$\mathbf{f}_{\mathbf{P R} 2}=-\left(\frac{B}{d^{2}}\right)\left(\frac{\mathbf{V}_{\mathbf{b}} \cdot \mathbf{r}_{\mathbf{b}}}{d^{2} c}\right) \mathbf{d}$

which is zero for the assumed circular orbit about the Sun, because $\mathbf{V}_{\mathbf{b}}$ is perpendicular to $\mathbf{r}_{\mathbf{b}}$. The final term in (7) in which $\mathbf{V}_{\mathbf{b}}$ appears is

$\mathbf{f}_{\mathbf{P R} 3}=-\left(\frac{B}{d^{2}}\right)\left(\frac{\mathbf{V}_{\mathbf{b}} \cdot \mathbf{r}}{d^{2} c}\right) \mathbf{d}$

D. Vokrouhlický (private communication, 2013) has suggested that (23) may contain secular terms, so that there is a secular drag which contains $V_{b}$ to the first power. This is confirmed by the following argument. Assume that

$\mathbf{d} \approx \mathbf{r}_{\mathbf{b}}=r_{b}\left(\cos n_{b} t \hat{\mathbf{x}}+\sin n_{b} t \hat{\mathbf{y}}\right)$

where $t$ is time, $n_{b}$ is the mean motion of the body about the Sun, $d \approx r_{b}$, and

$\mathbf{V}_{\mathbf{b}} \approx V_{b}\left(-\sin n_{b} t \hat{\mathbf{x}}+\cos n_{b} t \hat{\mathbf{y}}\right)$

Also,

$$
\begin{aligned}
\mathbf{r}= & r\{[\cos \Omega \cos (\omega+f)-\cos I \sin \Omega \sin (\omega+f)] \hat{\mathbf{x}} \\
& +[\sin \Omega \cos (\omega+f)+\cos I \cos \Omega \sin (\omega+f)] \hat{\mathbf{y}}+[\sin I \\
& \times \sin (\omega+f)] \hat{\mathbf{z}}\}
\end{aligned}
$$

(e.g., Rubincam, 1987, p. 1291). There will be a drag from (23) if taking the dot product of $\left(\mathbf{V}_{\mathbf{b}} \cdot \mathbf{r}\right) \mathbf{r}_{\mathbf{b}}$ with the unit along-track unit vector $\hat{\mathbf{t}}$ as given by (17) produces secular terms. This is the case:

$$
\begin{aligned}
\left(\mathbf{V}_{\mathbf{b}} \cdot \mathbf{r}\right)\left(\mathbf{r}_{\mathbf{b}} \cdot \hat{\mathbf{t}}\right)= & -V_{b} r r_{b}\left[\cos I \cos ^{2} \Omega \sin ^{2}(\omega+f) \cos ^{2} n_{b} t+\cos I\right. \\
& \times \sin ^{2} \Omega \cos ^{2}(\omega+f) \cos ^{2} n_{b} t+\cos I \sin ^{2} \Omega \sin ^{2}(\omega \\
& \left.+f) \sin ^{2} n_{b} t+\cos I \cos ^{2} \Omega \cos ^{2}(\omega+f) \sin ^{2} n_{b} t\right] \\
& + \text { periodic terms }
\end{aligned}
$$

Averaging the above expression over one revolution of the body about the Sun and the particle about the body yields

$\left\langle\left(\mathbf{V}_{\mathbf{b}} \cdot \mathbf{r}\right)\left(\mathbf{r}_{\mathbf{b}} \cdot \hat{\mathbf{t}}\right)\right\rangle=-\left(V_{b} r r_{b} \cos I\right) / 2$

Using this in (23) shows that not all terms to the first power in $V_{b}$ vanish in the Poynting-Robertson effect. However, $V_{b} / c$ is multiplied in (23) by $r / d$. Table 1 shows that $r / d \sim V_{b} / c$ for the planets when $r \sim R$, and $R / d \ll V_{b} / c$ for NEAs and main belt asteroids, so that (23) is of magnitude $\left(V_{b} / c\right)^{2}$ or smaller. Hence (23) is tiny for most Solar System applications.

\section{Other Poynting-Robertson terms}

While there appear to be no significant $V_{b} / c$ contributions, the Sun does have other direct effects on Poynting-Robertson, in addition to its indirect effect of illuminating and heating the body that the particle orbits around. It is a $v / c$ effect, rather than a $V_{b} / c$ effect. This can be seen by examining the remaining terms in (7):

$$
\begin{aligned}
\mathbf{f}_{\mathrm{PR} 4} & =-\left(\frac{B}{d^{2}}\right) \frac{\mathbf{v}}{c} \\
\mathbf{f}_{\mathrm{PR} 5} & =-\left(\frac{B}{d^{2}}\right)\left(\frac{\mathbf{v} \cdot \mathbf{r}_{\mathbf{b}}}{d^{2} c}\right) \mathbf{d}
\end{aligned}
$$

and

$\mathbf{f}_{\mathrm{PR} 6}=-\left(\frac{B}{d^{2}}\right)\left(\frac{\mathbf{v} \cdot \mathbf{r}}{d^{2} c}\right) \mathbf{d}$

In (30) $\mathbf{v} \cdot \mathbf{r}=0$ for circular orbits; this leaves (28) and (29). There is Poynting-Robertson drag from (28) because the acceleration acts against the velocity vector. There is also drag from (29), as may be seen from the following argument. Once again assuming (24), noting that $\mathbf{v}=v \hat{\mathbf{t}}$, and taking the dot-product of (17) with $\mathbf{r}_{\mathbf{b}}$ yields

$$
\begin{aligned}
\mathbf{v} \cdot \mathbf{r}_{\mathbf{b}}= & v \hat{\mathbf{t}} \cdot \mathbf{r}_{\mathbf{b}} \\
= & v r_{b}\left[-\cos \Omega \sin (\omega+f) \cos n_{b} t-\cos I \sin \Omega \cos (\omega+f)\right. \\
& \times \cos n_{b} t+\left[-\sin \Omega \sin (\omega+f) \sin n_{b} t+\cos I \cos \Omega \cos (\omega\right. \\
& \left.+f) \cos n_{b} t\right]
\end{aligned}
$$

so that

$$
\begin{aligned}
v\left(\hat{\mathbf{t}} \cdot \mathbf{r}_{\mathbf{b}}\right)^{2}= & v\left[\cos ^{2} \Omega \sin ^{2}(\omega+f) \cos ^{2} n_{b} t+\cos ^{2} I\right. \\
& \times \sin ^{2} \Omega \cos ^{2}(\omega+f) \cos ^{2} n_{b} t+\sin ^{2} \Omega \sin ^{2}(\omega \\
& \left.+f) \sin ^{2} n_{b} t+\cos ^{2} I \cos ^{2} \Omega \cos ^{2}(\omega+f) \cos ^{2} n_{b} t\right] \\
& + \text { periodic terms }
\end{aligned}
$$

assuming $I$ and $\Omega$ do not change significantly over one revolution of the particle about the body or one revolution of the body about the Sun. Averaging the above expression over these revolutions give

$$
\begin{aligned}
\left\langle v\left(\hat{\mathbf{t}} \cdot \mathbf{r}_{\mathbf{b}}\right)^{2}\right\rangle= & \left(v r_{b}^{2} / 4\right)\left[\cos ^{2} \Omega+\cos ^{2} I \sin ^{2} \Omega+\sin ^{2} \Omega+\cos ^{2} I\right. \\
& \left.\times \cos ^{2} \Omega\right] \\
= & v r_{b}^{2}\left(1+\cos ^{2} I\right) / 4
\end{aligned}
$$

assuming no shadow passage. Thus (32) is nonzero and when used in (29) the resulting equation agrees with Burns et al. (1979), Goldreich and Tremaine (1982), Mignard (1984), Vokrouhlický et al. (2007), and Bottke et al. (2013).

\section{Discussion}

The Sun shines on the particle as the body orbits the Sun with speed $V_{b}$. The question addressed here is whether the Sun shining directly on the particle produces a significant Poynting-Robertson effect on the particle's orbit of order $V_{b} / c$. Any $V_{b} / c$ effect would not be particularly important for particles orbiting Jupiter or Saturn where $V_{b} \sim v$. But the effect would be large for the inner planets, where $V_{b} \sim 5 v$, and gigantic for NEAs, where $V_{b} \sim 3 \times 10^{4} v$ (last column of Table 1 ).

It was shown above that, for spherical bodies and for circular orbits of the particle and body, that there are no significant terms of order $V_{b} / c$. This was demonstrated from the point of view of the Sun's rest frame and from the frame traveling with the body. As (27) shows, there are Poynting-Robertson terms with $V_{b}$ to the first power in them, but these are multiplied by $r / d$, which tends to be $\ll 1$ in the Solar System. The ratio $r / d$ is particularly small for main belt asteroids and NEAs (Table 1). Hence there is no huge Poynting-Robertson effect from $V_{b} / c$ on particles orbiting asteroids for the stated assumptions.

A fuller treatment would include eccentric orbits and relaxing the assumption that the change in the position of the body in its orbit about the Sun is negligible over one revolution of the particle about the body, and that $\Omega$ and $I$ are fixed. Further, the Sun is not a point source of light, and Solar System bodies are not perfect 


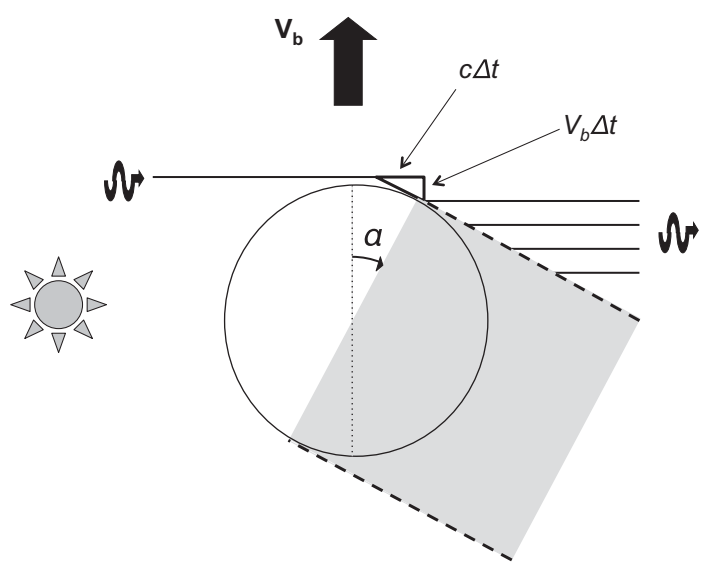

Fig. 6. The terminator and shadow of the spherical body as seen in the Sun's rest frame. A photon (wavy arrow) on a streamline (left horizontal line) grazes the body, which is moving up with speed $V_{b}$ to meet it. $\alpha$ is the angle of the terminator. The lower rightmost horizontal lines mark photon streamlines, the rears of which mark the edge of the body's shadow.

spheres, particularly asteroids. However, these considerations seem unlikely to change the main result.

The framework of the present paper is Newtonian (Burns et al. 1979). There may well be contributions of order $\left(V_{b} / c\right)^{2}$ to Poynting-Robertson. Any investigation at the $\left(V_{b} / c\right)^{2}$ level would entail the full machinery of special relativity, as the Lorentz transformations are of this order. One suspects this would be very involved.

\section{Acknowledgments}

I thank Joseph A. Burns and David Vokrouhlický for correcting mistakes and making insightful comments which greatly improved the manuscript. The support of the NASA Advanced Exploration Systems and SALMON proposals is gratefully acknowledged.

\section{Appendix A}

The terminator on the body can be found from the viewpoint of the Sun's rest frame with the aid of Fig. 6. Slices of the spherical body parallel to the body's orbit plane will be circles, one of which is shown in the figure. Assume photons from the Sun come in on parallel streamlines. The body is moving up in the figure. Let the last photon which grazes the leading side at a particular instant be one which travels on the streamline shown in the figure (left upper horizontal line); all of the photons following behind on that streamline will strike the sunny portions of the body as the body cuts across the streamline. Thus the last photon marks the terminator.

Perhaps the easiest way to find the terminator is by going backward in time by $\Delta t$ before the graze. The place on the body where the photon grazes will be moving down and travel a distance $V_{b} \Delta t$ from the graze; the photon will be traveling horizontally at speed $c$ and cover a distance $c \Delta t$. The small right triangle with sides $V_{b} \Delta t$ and $c \Delta t$ must be situated where the hypotenuse is tangent to the circle; this place is at an angle $\alpha$, where $\tan \alpha=\left(V_{b} \Delta t\right) /(c \Delta t)=V_{b} /$ $c$. If the graze point were any further around the circle than $\alpha$, then going back further in time the photon would have to travel through the body to make it back to the Sun.

That the body's shadow is rotated by an angle $\alpha$ can also be seen with the aid of Fig. 6. Photons that were on the streamline ahead of the grazing photon made it past the body, so that the streamline is full of photons from the terminator to the indefinite right. The last grazing photon that was on a streamline at time $\delta t$ before was at a position $V_{b} \delta t$ below and has traveled a distance $c \delta t$ to the right. No photons follow it, being blocked by the body, so the shadow begins there. Likewise, the last grazing photon at time $2 \delta t$ before was on a streamline $2 V_{b} \delta t$ below and traveled $2 c \delta t$ to the right, with no photons following, and so on. The trailing edge of these preceding streamlines (rightmost horizontal lines) is marked by a dashed line in Fig. 6 and delineate the edge of the shadow. The dashed line can be shown to be at an angle $\alpha$ with the horizontal, where once again $\tan \alpha=V_{b} / c$.

\section{References}

Blanco, V.M., McCuskey, S.W., 1961. Basic Physics of the Solar System. AddisonWesley, Reading, Mass

Bottke, W.F., Vokrouhlický, D., Nesvorný, D., Moore, J.M., 2013. Black rain: The burial of the Galilean satellites in irregular satellite debris. Icarus 223, 775-797. http://dx.doi.org/10.1016/j.icarus.3013.01.008.

Burns, J.A., Lamy, P.L., Soter, S., 1979. Radiation forces on small particles in the Solar System. Icarus $40,1-48$

Burns, J.A., Showalter, M.R., Morfill, G.E., 1984. The ethereal rings of Jupiter and Saturn. In: Greenberg, R., Brahic, A. (Eds.), Planetary Rings. Univ. Arizona Press, Tucson, pp. 200-272.

Burns, J.A. et al., 1999. The formation of Jupiter's faint rings. Science 284, 1146 1150. http://dx.doi.org/10.1126/science.284.5417.1146.

Goldreich, P., Tremaine, S., 1982. The dynamics of planetary rings. Annu. Rev. Astron. Astrophys. 20, 249-283. http://dx.doi.org/10.1146 annurev.aa.20.090182.001341.

Horanyi, M., Burns, J.A., 1991. Charged dust dynamics: Orbital resonance due to planetary shadows. J. Geophys. Res. 96, 19283-19289. http://dx.doi.org/ 10.1029/91JA01982.

Jacobson, S.A., Scheeres, D.J., 2011. Dynamics of rotationally fissioned asteroids: Source of observed small asteroid systems. Icarus 214, 161-178. http:// dx.doi.org/10.1016/j.icarus.2011.04.009.

Kopp, G., Lean, J.L., 2011. A new, lower value of total solar irradiance: Evidence and climate significance. Geophys. Res. Lett. 38, L01706. http://dx.doi.org/10.1029/ 2010 GL045777.

Mignard, F., 1984. Effects of radiation forces on dust particles. In: Greenberg, R. Brahic, A. (Eds.), Planetary Rings. Univ. Arizona Press, Tucson, pp. 333-366.

Paddack, S.J., 1969. Rotational bursting of small celestial bodies: Effects of radiation pressure. J. Geophys. Res. 74, 4379-4381.

Poynting, J.H., 1903. Radiation in the Solar System; its effect on temperature and its pressure on small bodies. Mon. Not. R. Astron. Soc. 72 (Appendix), 265-266.

Richardson, D.C., Bottke, W.F., Love, S.G., 1998. Tidal distortion and disruption of Earth-crossing asteroids. Icarus 134, 47-76. http://dx.doi.org/10.1006/ icar.1998.5954.

Robertson, H.P., 1937. Dynamical effects of radiation in the Solar System. Mon. Not. R. Astron. Soc. 97, 423-437.

Rubincam, D.P., 1987. LAGEOS orbit decay due to infrared radiation from Earth. J. Geophys. Res. 92, 1287-1294.

Rubincam, D.P., 2000. Radiative spin-up and spin-down of small asteroids. Icarus $148,2-11$.

Sommerfeld, A., 1964. Optics. Academic Press, New York.

Vokrouhlický, D., Nesvorný, D., Dones, L., Bottke, W.F., 2007. Thermal forces on planetary ring particles. Astron. Astrophys. 471, 717-730. http://dx.doi.org/ 10.1051/0004-6361:20067029. 